\title{
The Efficacy of Warm Compresses in the Treatment of Meibomian Gland Dysfunction and Demodex Folliculorum Blepharitis
}

Orla Murphy

Technological University Dublin, orla.murphy@tudublin.ie

Veronica O'Dwyer

Technological University Dublin, veronica.odwyer@tudublin.ie

Aoife Lloyd-Mckernan

Technological University Dublin

Follow this and additional works at: https://arrow.tudublin.ie/scschphyart

Part of the Ophthalmology Commons, and the Optometry Commons

\section{Recommended Citation}

Orla Murphy, Veronica O' Dwyer \& Aoife Lloyd-Mckernan (2020) The Efficacy of Warm Compresses in the Treatment of Meibomian Gland Dysfunction and Demodex Folliculorum Blepharitis, Current Eye Research, 45:5, 563-575, DOI: 10.1080/02713683.2019.1686153

This Article is brought to you for free and open access by the School of Physics \& Clinical \& Optometric Science at ARROW@TU Dublin. It has been accepted for inclusion in Articles by an authorized administrator of ARROW@TU Dublin. For more information, please contact arrow.admin@tudublin.ie, aisling.coyne@tudublin.ie, gerard.connolly@tudublin.ie.

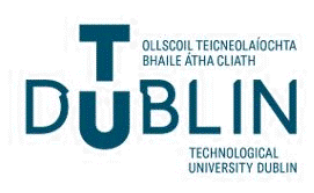




\section{The Efficacy of Warm Compresses in the Treatment of Meibomian Gland Dysfunction and Demodex Folliculorum Blepharitis}

\section{Orla Murphy, Veronica O’ Dwyer \& Aoife Lloyd-Mckernan}

To cite this article: Orla Murphy, Veronica O' Dwyer \& Aoife Lloyd-Mckernan (2020) The Efficacy of Warm Compresses in the Treatment of Meibomian Gland Dysfunction and Demodex Folliculorum Blepharitis, Current Eye Research, 45:5, 563-575, DOI: 10.1080/02713683.2019.1686153

To link to this article: https://doi.org/10.1080/02713683.2019.1686153

\section{曲 Published online: 19 Nov 2019.}

Submit your article to this journal $\sqsubset$

Џll Article views: 452

Q View related articles 두

View Crossmark data $₫$

Citing articles: 6 View citing articles $[7$ 


\title{
The Efficacy of Warm Compresses in the Treatment of Meibomian Gland Dysfunction and Demodex Folliculorum Blepharitis
}

\author{
Orla Murphy $\mathbb{1}$, Veronica O' Dwyer, and Aoife Lloyd-Mckernan \\ School of Physics \& Clinical \& Optometric Sciences, Dublin Institute of Technology, Dublin, Ireland
}

\begin{abstract}
Purpose: To investigate and compare the effect of warm compresses on meibomian gland dysfunction and Demodex folliculorum blepharitis.

Methods: Forty-two subjects (13 males, 29 females; mean age of 56.45 years) enrolled and completed the two-month warm compress treatment study. Three warm compress therapies were compared: Warm face cloth, MGDRx EyeBag® and OPTASE ${ }^{\mathrm{TM}}$ Moist Heat Mask. Subjects attended for four visits: baseline, two weeks, four weeks, and eight weeks. Subjective symptoms, osmolarity, non-invasive tear break-up time, ocular surface staining, Schirmer I test, meibum expressibility and clarity, and eyelash manipulation and epilation to assess for the presence of Demodex folliculorum, were measured at each visit.

Results: Meibomian gland dysfunction, based on a composite score of meibum quality and expressibility, reduced significantly with the MGDRx EyeBag ${ }^{\circledR}$ and the $\operatorname{OPTASE}^{\mathrm{TM}}$ Moist Heat Mask $(p<.05)$. There was no significant difference in efficacy for treating meibomian gland dysfunction between the two devices $(p=.29)$. No improvement in meibomian gland dysfunction was detected with the warm face cloth. Only the OPTASE $^{\text {TM }}$ Moist Heat Mask significantly reduced the quantity of Demodex folliculorum over eight-weeks of treatment ( $p=.036$, only baseline to week eight significant $p=.008$ ). Symptoms and ocular surface staining improved significantly in all three groups $(p<.05)$. There was no significant change observed in osmolarity, non-invasive tear break-up time or Schirmer I test within each group ( $p>.05$, respectively).

Conclusion: The MGDRx EyeBag® and the OPTASE ${ }^{\text {TM }}$ Moist Heat Mask exhibited superior efficacy in treating signs and symptoms of meibomian gland dysfunction, compared to the use of a warm face cloth, over the eight-week period. The OPTASE ${ }^{\mathrm{TM}}$ Moist Heat Mask demonstrated dual therapeutic abilities, treating both meibomian gland dysfunction and Demodex folliculorum blepharitis. Repeated application of heat for the treatment of meibomian gland dysfunction may continue to present a good home-remedy option for patients.
\end{abstract}

\section{ARTICLE HISTORY}

Received 12 December 2018 Revised 14 October 2019

Accepted 22 October 2019

\section{KEYWORDS}

Demodex folliculorum; Blepharitis; Meibomian gland dysfunction; warm compress; treatment

\section{Introduction}

The meibomian glands are a group of holocrine glands found in the upper and lower eyelids. They consist of parallel rows of small secretory acini arranged around a larger central duct which opens onto the eyelid margin. ${ }^{1,2}$ The role of the meibomian glands is to supply meibum to the ocular surface: to prevent evaporation of the tear film, provide a smooth refractive corneal surface for better vision, and act as a barrier to microbial agents and organic matter such as dust. ${ }^{3-6}$ Disruption to this supply, often through terminal duct obstruction or changes in glandular secretion, can interfere with the homeostasis of the tear film and ocular surface; leading to inflammation and subsequent symptoms of discomfort. ${ }^{5,7}$

The eyelash follicles are located within the eyelids, anterior to the meibomian glands. Because of their close proximity to oneanother, anomalies of the eyelash follicles and the meibomian glands are often seen in combination with each other. ${ }^{8-11}$ One such anomaly, is the inhabitation of the eyelashes and meibomian glands by the common ecto-parasites: Demodex folliculorum (DF) and Demodex brevis (DB). Demodex folliculorum tend to reside in clusters in the eyelash follicles ${ }^{12-14}$ and have been most commonly linked with anterior blepharitis ${ }^{12,15-19}$ while DB generally burrow deeper into the meibomian glands and have been more commonly associated with meibomian gland dysfunction (MGD). ${ }^{13,20-22}$

The association between DB infestation and severe MGD and keratitis has been described in the literature. ${ }^{10,23}$ Although DF are generally associated with anterior blepharitis and infestation of the eyelash follicles, the prevalence of DF in the eyelash follicles of MGD patients has been reported to vary between $46.5 \%$ to $85 \%{ }^{24,25}$ Treatment in the form of lid scrubs, targeted at reducing the quantity of both DB and DF, has shown improvement in ocular surface health and patient symptoms. ${ }^{10,23}$

The mainstay treatment recommended for DF and DB infestation is lid scrubs with diluted quantities of tea tree oil. ${ }^{26-28}$ While lid scrubs have been indicated as an early treatment option for patients with mild MGD, warm compress therapy remains the principal treatment for MGD. ${ }^{29}$ In order for warm compresses to be effective, heat must pass through the anterior eyelid structures, including the eyelashes, to warm and melt thickened meibum within the meibomian glands. The melting temperature of normal meibum is approximately $32^{\circ} \mathrm{C}$, and is higher at approximately $35^{\circ} \mathrm{C}$ in obstructed glands with altered secretions. ${ }^{29,30}$ Therefore, it is recommended that for warm compresses to be effective at 
treating MGD, they need to heat the inner eyelid to a temperature of $\geq 40^{\circ} \mathrm{C} .{ }^{31}$ However, both DB and DF prefer lower temperatures, and Zhao et al. have shown that temperatures above $37^{\circ} \mathrm{C}$ are damaging to $\mathrm{DF} .^{32}$ Higher temperatures cause death by protein coagulation and denaturation, and eventual paralysis of the DF nervous system. ${ }^{32}$ Murakami et al. have shown that although there is variety in inner eyelid temperatures achieved by different warm compresses, most methods do manage to reach outer eyelid temperatures of $\geq 40^{\circ} \mathrm{C}$. ${ }^{33}$ Hence, as heat from the warm compress passes through the eyelash follicles to heat the inner eyelid, it could potentially have the effect of killing the DF within the eyelash follicle. Intense pulsed light used for treating MGD has shown therapeutic ability for Demodex infestation. ${ }^{34}$

Traditionally, home based warm compresses were carried out using a warm face cloth. ${ }^{29,35}$ However, this method has its limitations, including poor heat retention, ${ }^{36}$ and inconvenience leading to reduced compliance. ${ }^{29}$ Over the years, more patient-friendly warm compresses have become available, such as the MGDRx EyeBag® (The EyeBag® Company, Halifax, UK) and the OPTASE ${ }^{\mathrm{TM}}$ Moist Heat Mask (Scope Ophthalmics Ltd., Dublin, Ireland). While both warm compresses are principally very similar; they are heated in a microwave, and a single heating is required to provide 10 minutes of therapy; there are fundamental differences between them. The OPTASE ${ }^{\mathrm{TM}}$ Moist Heat Mask contains HydroBead $^{\mathrm{TM}}$ Technology, which absorbs moisture from the air, and when heated, releases it to provide a moist heat. The moist heat therapy helps to soften eyelash debris in patients with anterior blepharitis, and restores moisture to the eye and surrounding area, in conjunction with improving meibum flow, tear film quality and reduced tear film evaporation. By contrast, the MGDRx EyeBag® is filled with flax seed and provides a dry heat when applied to the eyelids. Manufacturers recommend it for relief of, including but not limited to; MGD, blepharitis, dry eye syndrome, and rosacea. Their efficacy in the treatment of Demodex blepharitis has not previously been investigated.

The aim of the current study was to assess the therapeutic effect of these common home-based warm compresses on DF infestation in MGD patients.

\section{Materials and methods}

\section{Study design}

This was a single-centre, interventional, randomised, controlled, examiner masked clinical trial. The study was conducted under the Tenets of Helsinki Declaration of Human Studies ${ }^{37}$ and approved by the Technological University Dublin Research Ethics Committee. All subjects were recruited through the National Optometry Centre, Technological University Dublin, Ireland's private and student optometry clinics. Written informed consent was obtained from all subjects prior to enrolment. Subjects were examined for the presence of MGD and DF and were given a warm compress to use for 10 minutes twice a day for two weeks, followed by 10 minutes once a day for the remaining six weeks. The frequency and duration of treatment was based around MGDRx EyeBag® and OPTASE $^{\mathrm{TM}}$ Moist Heat Mask manufacturers guidelines on instructions for use,
10 minutes twice a day for the first two weeks and then reduce frequency. Instructions were kept consistent for all treatment groups. As DF have a lifespan of two-three weeks, ${ }^{38}$ an eight week treatment duration was decided to ensure treatment was provided over generations of DF.

\section{Study participants}

A priori analysis for repeated measures ANOVA $(\alpha=0.05$, $1-\beta=0.80$, effect size $=0.5: 3$ groups, 2 measurements) was used to calculate the minimum sample size required. Effect size was calculated from the mean and standard deviation of the difference of DF presentation on lash manipulation and microscopic examination from previous data collected on 428 eyelashes: $0.84 / 1.59=0.52$. The minimum total sample size required was 33 subjects; 11 subjects per group. Fifty subjects in total were enrolled between April 2017 and May 2018. Subjects were eligible to participate if they were; $\geq 18$ years of age and had $\geq$ G1 MGD based on meibomian gland expression according to the diagnostic subcommittee of the International Workshop on Meibomian Gland Dysfunction. ${ }^{39}$ Subjects were excluded if they; wore contact lenses, were pregnant, had a systemic disease or were using topical/systemic medication known to affect the eyes, presented with ocular disease (with the exception of MGD and blepharitis), were currently using MGD/blepharitis treatment or had used such treatment within the last six months, or had ocular surgery in the last six months.

\section{Study procedures}

Subjects attended the National Optometry Centre for four visits in total: Baseline, week two, week four, and week eight. All examinations were conducted in the same room, at the same time of day ( \pm 30 minutes), by the same examiner (author OM). Examinations were conducted in the same order at each visit, from least invasive to most invasive. ${ }^{40,41}$

Subjects completed a validated, modified OSDI symptom questionnaire, ${ }^{42}$ and were scored from 0-100, with higher numbers indicating increased severity of symptoms. The modified OSDI questionnaire was chosen as it includes questions that are more specific to Demodex blepharitis: itchy eyes. Modification of existing questionnaires has been previously recommended in order to increase the sensitivity of the questionnaire in diagnosing MGD and blepharitis, ${ }^{39}$ and modification of the OSDI questionnaire has been previously used in Demodex studies. $^{28,43}$ Non-invasive tear break-up time (NITBUT) was measured using the Medmont E300 Corneal Topographer (Medmont International Pty Ltd., Victoria, Australia). An average of three measurements was recorded for each eye. Tear osmolarity was measured using the TearLab Osmolarity System (TearLab Corporation, San Diego, California). One measurement was taken from each eye. An inter-eye difference of $>8 \mathrm{mOsm} / \mathrm{L}$ indicates tear film instability. ${ }^{44}$ A cut-off threshold measurement of $>308$ $\mathrm{mOsm} / \mathrm{L}$ is most sensitive to differentiating between normal and mild to moderate dry eye subjects. ${ }^{4,45}$ The eye with the highest tear osmolarity measurement at baseline was chosen as the study eye, and this eye was used for all data analysis. 
Ocular surface staining was assessed using fluorescein dye (Fluorets; Chauvin Pharmaceuticals, United Kingdom) and a yellow wrattan filter, and graded using the Oxford Grading System. ${ }^{46}$ Corneal, and nasal and temporal bulbar conjunctival staining were each graded individually on a 6 point scale $(0-5$ for each location, 0 indicating no staining, 5 indicating severe staining). Results were then combined to provide a composite score $(0-15)$ for each eye. ${ }^{46}$ Schirmer I test (without anaesthesia) (Tear Flo; HUB Pharmaceutical, United Kingdom) was performed to measure subjects tear secretion. Subjects were asked to close their eyes, ${ }^{47}$ and the score was measured as the wetting length in $\mathrm{mm} / 5 \mathrm{~min}$. Subjects were considered to have 'dry eye' if found to have three or more of the following six parameters; OSDI $\geq 12$, osmolarity $\geq 308 \mathrm{mOsm} / \mathrm{L}$, tear osmolarity variance $\geq$ $8 \mathrm{mOsm} / \mathrm{L}$, NITBUT $<10$ secs, ocular surface staining $\geq 3$ Oxford score or Schirmer I score $\leq 5 \mathrm{~mm} / 5 \mathrm{~min}$.

Slit-lamp bio-microscopy (Topcon SL-D701, Topcon Medical Systems Inc., Dublin, Ireland) was conducted to examine the meibomian glands in accordance with the diagnostic subcommittee of the International Workshop on Meibomian Gland Dysfunction $^{39}$; firm digital pressure was applied to the eyelid margins and the number of glands expressible, from the central eight glands, was graded on a four-point scale $(0=$ all glands expressible, $1=3-4$ glands expressible, $2=1-2$ glands expressible, 3 = no glands expressible). The quality of meibum secreted was graded on a four-point scale ( $0=$ clear, $1=$ cloudy, $2=$ cloudy with particulate, $3=$ thick like toothpaste). As recommended, composite scores derived from the expression of both upper and lower eyelids was generated and used for statistical analysis. ${ }^{39}$ Lastly, one eyelash from each eyelid was examined for the presence of DF through eyelash rotation and epilation on the slit-lamp bio-microscope. Examiners chose to examine one eyelash per eyelid at each visit as subjects were being examined over four visits. Many of the subjects were older, some with chronic MGD and blepharitis, and therefore had a reduced number of eyelashes. Hence, many subjects were not keen on having any eyelashes removed at each visit. In an effort to increase subject participation and retention, it was decided to only remove one eyelash from each lid at each visit. Eyelashes were chosen in accordance with the modified Coston method. ${ }^{48}$ Briefly, the modified Coston method recommends selectively choosing eyelashes in an effort to maximise potential of finding $\mathrm{DF}^{48}$ As such, eyelashes with cylindrical dandruff or mis-directed eyelashes (possibly due to a damaged follicle) were chosen. In the absence of cylindrical dandruff or mis-directed eyelashes, eyelashes were chosen from the central region of the eyelid. In keeping with the modified Coston method, alcohol and fluorescein were used to facilitate imaging of the mites on microscopic examination. A limitation of the modified Coston method is that it can lead to undercounting; through DF remaining within the follicle after the eyelash has been removed. It has been established previously that DF can be detected through eyelash rotation, ${ }^{49}$ and a recent study has also shown that the quantity of DF detected on rotation of the eyelash within the follicle is a better indicator of severity of infestation..$^{50}$ As such, both quantity of DF found on eyelash rotation and on microscopic examination have been examined in this study. Each eyelash was rotated in situ, using sterile forceps, to examine for the presence of DF within the follicle. The number of DF tails seen emerging from the eyelash follicle was counted. The same eyelash was then epilated and examined on a microscope slide using the modified Coston method, ${ }^{48}$ and the number of DF present was recorded. Similar to MGD, a composite score was derived for each eye from quantities found on upper and lower eyelids and used for statistical analysis. The percentage of subjects with DF in each group was also determined. Positive DF infestation was defined as the presence of $\geq 1$ DF detected on either eyelash manipulation or microscopic examination. Based on work by Randon et al. the proportion of DF was further classified into non-pathogenic ( $\leq 3$ mites) and pathogenic infestation ( $>4$ mites) per eye. ${ }^{22}$ Quantities of Demodex folliculorum detected on eyelash manipulation were used for this classification.

The intra-class correlation co-efficient (ICC) was determined to examine the agreement in pre-treatment results between the right and left eyes for each participant. A twoway mixed analysis with absolute agreement and $95 \%$ confidence intervals was conducted. ${ }^{51}$ Quantity DF (ICC: 0.71), MGD Grade (ICC: 0.93), osmolarity (ICC:0.68), NITBUT (ICC:0.82), ocular surface staining (ICC: 0.77), Schirmer I test (ICC: 0.91 ) and dry eye (ICC:0.67). Values of less than 0.5 indicate 'poor' agreement, between 0.5 and 0.75 'moderate' agreement, between 0.75 and 0.9 'good' agreement, and greater than 0.90 'excellent' agreement. ${ }^{51}$ As recommended, data analysis was conducted on one eye only for each participant. ${ }^{52}$ As all correlations were between moderate to excellent, either eye was considered eligible for selection. Therefore, the eye selected for data analysis was chosen based on the higher tear osmolarity value at baseline. This is in keeping with previous studies and osmolarity measurement guidelines. ${ }^{4,45,53}$

Following enrolment, subjects were randomly allocated one of three treatments to use at home: Face cloth (Group 1, conventional treatment: $\mathrm{n}=12$ ), MGDRx EyeBagß (Group 2, dry heat: $\mathrm{n}=16$ ), OPTASE ${ }^{\mathrm{TM}}$ Moist Heat Mask (Group 3, moist heat: $\mathrm{n}=14$ ). In keeping with manufacturers' guidelines, each subject was given an instruction leaflet and was required to use the treatment for 10 minutes twice a day for the first two weeks. Frequency of compresses was reduced to 10 minutes once a day from weeks three to eight. Subjects in Group 1 were instructed to pour $200 \mathrm{ml}$ of boiled water into a bowl and allow it to cool for 10 minutes before beginning treatment. This created a water temperature ranging from $50^{\circ} \mathrm{C}$ to $39^{\circ} \mathrm{C}$ over the 10 minute treatment time (tested using a HYGIPLAS Easy temperature pocket catering thermometer and porcelain bowl). Subjects' were then required to re-heat the face cloth every two minutes, by immersing it in the same bowl of cooled, boiled water; to maintain temperature at therapeutic levels. ${ }^{31,54}$ Groups 2 and 3 were instructed to heat their compress in the microwave for 15-30 seconds depending on the power of their microwave, as per manufacturers' guidelines.

\section{Statistical analysis}

Statistical analysis was performed using SPSS IBM (ver. 25.0). Normality was measured using Shapiro-Wilk statistical test. Repeated measures Analysis Of Variance (ANOVA) with Bonferroni post-hoc analysis was used to analyse repeated measures within each group over time for parametric data. Friedman's test was used to analyse repeated measures, within 
each group, across different visits for non-parametric data. With Friedman's test, post-hoc analysis was conducted, where appropriate, using Wilcoxon signed ranks test for pairwise comparisons, adjusted using Bonferroni correction ( $\alpha=0.05 /$ number of comparisons: $\alpha=0.05 / 6=0.0083) .{ }^{55}$ One way ANOVA with Bonferroni post-hoc analysis was used to analyse data between continuous variables at baseline and at different visits for parametric data. Kruskal Wallis (K-W) was used for non-parametric data. With K-W, post-hoc analysis was conducted, where appropriate, using Mann Whitney- $U$ test for pairwise comparison, adjusted using Bonferroni correction ( $\alpha=0.05 /$ number of comparisons: $\alpha=0.05 / 3=0.0167) .{ }^{55}$ Parametric data was expressed as mean \pm standard deviation, non-parametric data was expressed as median and inter-quartiles. Post-hoc analysis was only conducted on significant findings. A $p$-value $<0.05$ was considered statistically significant, with the exception of Bonferroni adjusted post-hoc analysis as described above.

\section{Results}

Fifty subjects were enrolled between April 2017 and May 2018. Attrition rate was $16 \%$. Four subjects withdrew from the study, without any known adverse events and were lost to follow up. Two subjects discontinued as they felt their symptoms were worsening. A further two were removed from data analysis as their records were incomplete. Following attrition, 42 subjects ( 13 males and 29 females) with a mean age of $56.45 \pm 16.71$ years completed the two-month warm compress treatment study. At baseline, an overall proportion of $57.1 \%$ DF was found, with a mean quantity of 2.31 (range $0-13$ ) and 1.14 (range $0-9$ ) mites on lash rotation and microscopic examination respectively. There was no significant difference in age (Group 1: $59.08 \pm 14.27$, Group 2: $58.13 \pm 15.83$, Group 3: $52.29 \pm 19.78, \mathrm{~K}-\mathrm{W}: p=.754)$ or quantity of $\mathrm{DF}$ on either lash rotation or microscopic examination (K-W: $p=.784$ and $p=.847$ ) between the three groups before treatment.

\section{Dry eye disease}

Applying the dry eye classification previously outlined in Study Procedures, the proportion of subjects with dry eye in each group, at each visit, is shown in Figure 1. No significant change in proportion of subjects with dry eye was detected in any of the three treatment groups post-treatment (Group 1-3: Friedman's $p=.362, p=.768$ and $p=.284$, respectively).

\section{Demodex folliculorum analysis}

Table 1 displays the quantity of DF at each visit for each treatment group. Figure 2 illustrates the change in quantity of DF detected on eyelash rotation within each group over the eight weeks. Within treatment analysis showed that the quantity of DF dropped significantly over the duration of the study in Group 3 (OPTASE $^{\text {TM }}$ Moist Heat Mask) (Friedman's $p=.036$ ). Post-hoc pairwise comparisons using Wilcoxon signed ranks test, with alpha level adjusted for Bonferroni correction, showed only the change from baseline to week eight to be significant (median: 1.50, IQR: 0-4.25 versus median: 0, IQR: 0-1.5; WSR $p=.008$ ). There was no significant change in DF quantity in Group 1 (Warm Face Cloth) or Group 2 (MGDRx EyeBag®) over the eight weeks (Friedman's $p=.884$ and $p=.661$, respectively). Between treatments analysis did not show any significant difference between the treatments over the eight weeks (K-W $p>.05$, Table 1).

The median and interquartile values, for quantity of DF detected on microscopic examination at each visit, for each group, are also shown in Table 1. In contrast to results observed on eyelash rotation, there was no significant change in DF quantity detected on microscopic examination over time in each group (Friedman's $p>.05$ ) or between treatments $(\mathrm{K}-\mathrm{W} p>.05)$.

\section{Dry Eye Disease}

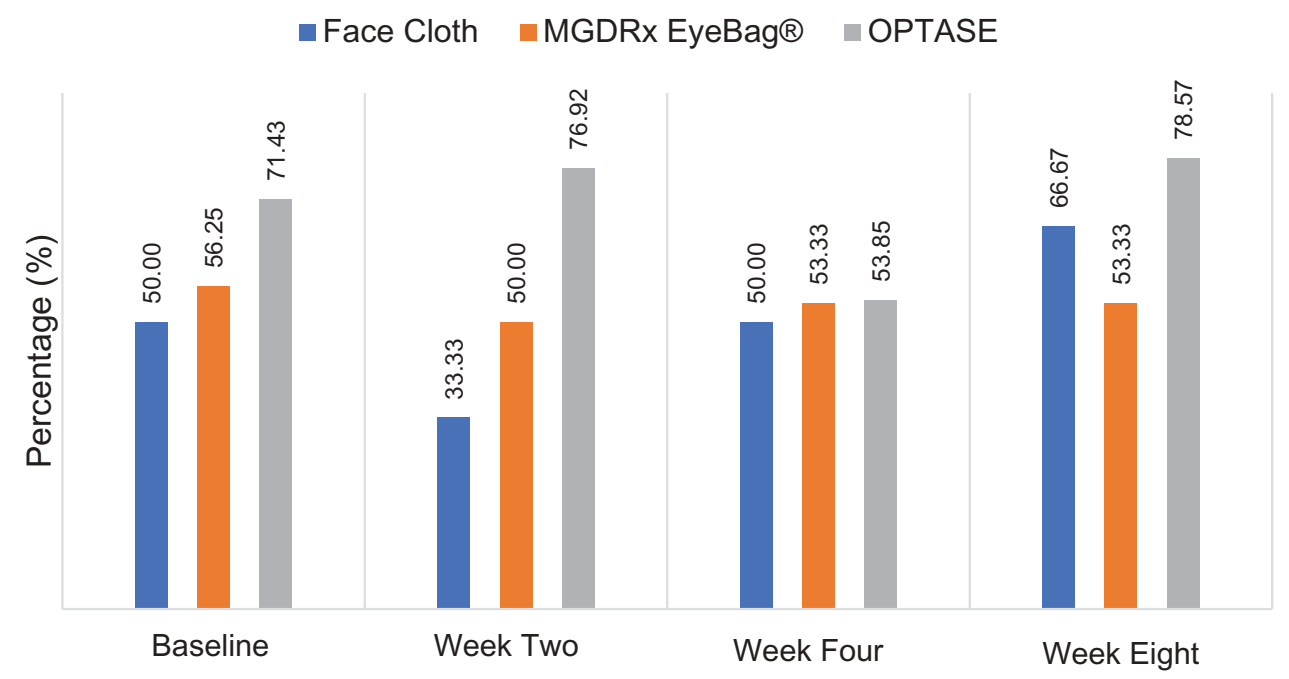

Figure 1. Proportion of subjects with dry eye disease in each warm compress treatment group at each time point. 
Table 1. Demodex folliculorum quantity (median and interquartile range) before and after treatment.

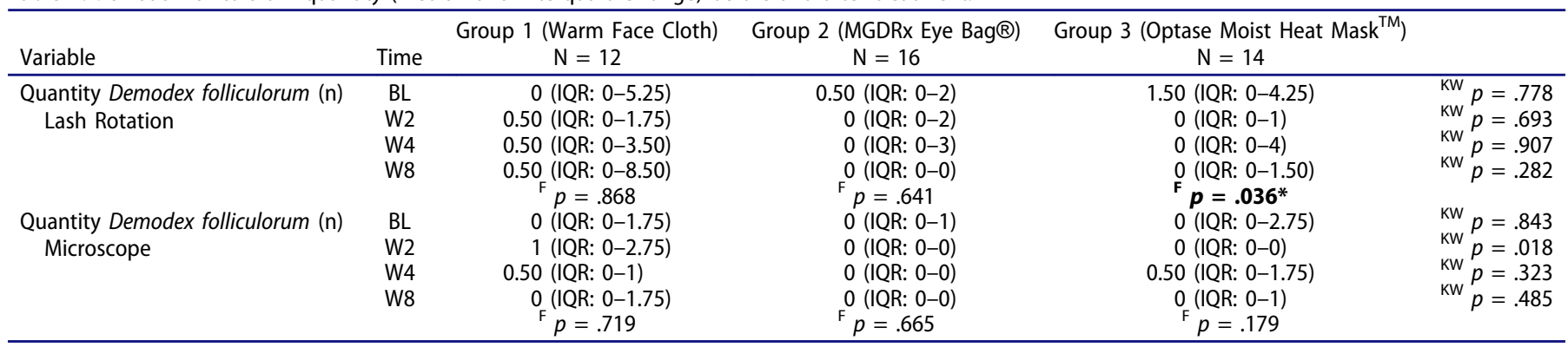

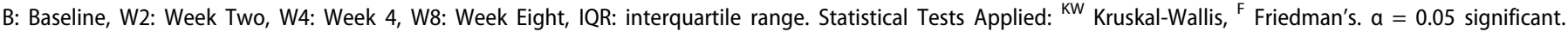
*Significant results highlighted in bold.

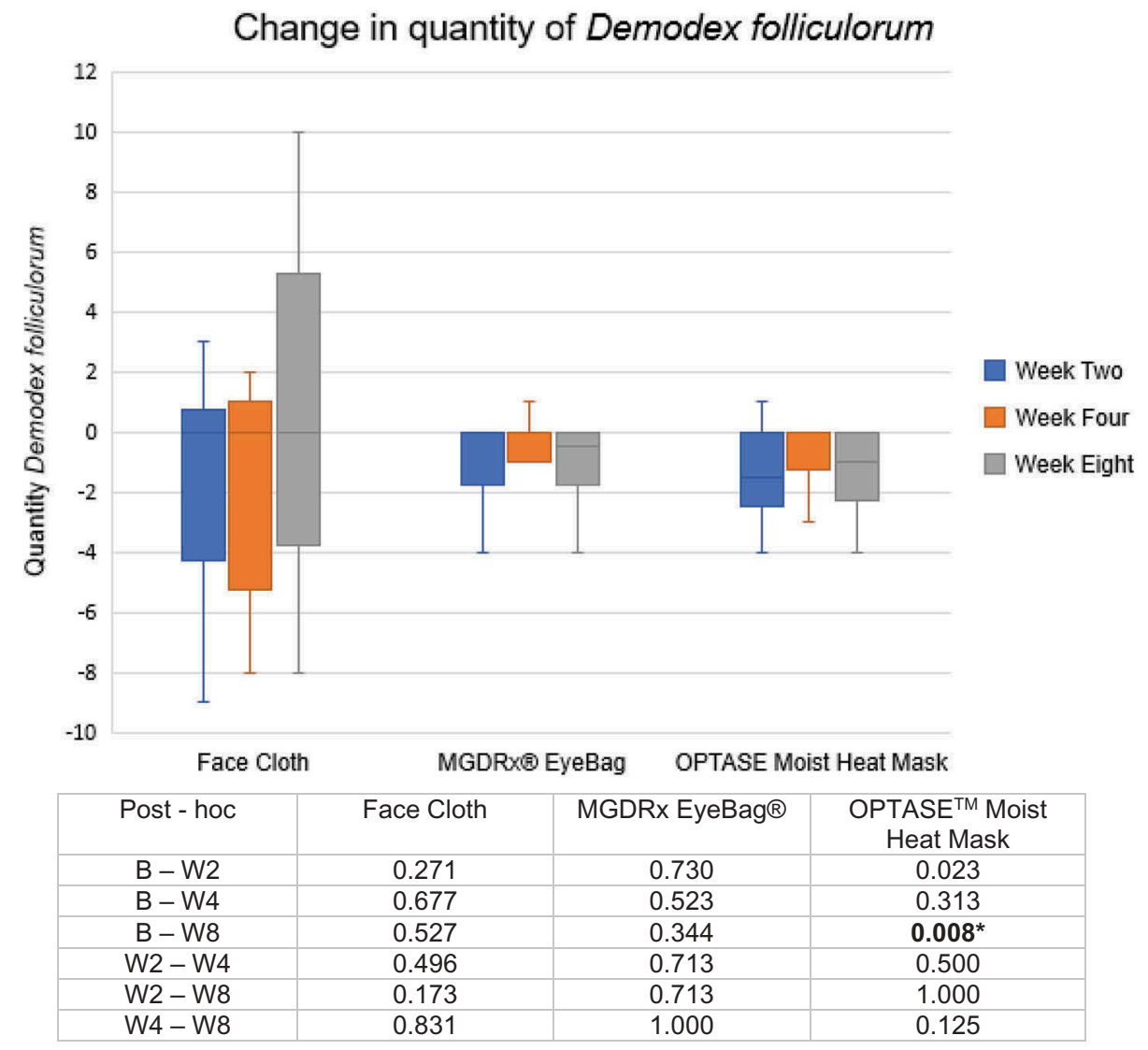

Post - hoc analysis: Wilcoxon signed ranks test pairwise comparison with Bonferroni adjusted correction applied ( $\alpha \leq 0.008$ significant). B: Baseline, W2: Week Two, W4: Week 4, W8: Week Eight. *Significant results highlighted in bold.

Figure 2. Box plot illustrating the change in quantity of Demodex folliculorum at two, four, and eight weeks, with each treatment.

The overall proportion of subjects with DF in each group at baseline was: Group 1-66.67\%, Group 2-50.00\%, and Group 3-57.14\%. There was no significant difference found between the groups at baseline (K-W $p=.609)$. This reduced to an overall proportion of: Group 1-58.33\%, Group $2-25.00 \%$ and Group 3-50.00\% after eight weeks. Table 2 shows the proportion of subjects with pathogenic and nonpathogenic infestation for each treatment group, at each point in time. Group 3 demonstrated the largest reduction in pathogenic infestation of the three groups $(-14.29 \%, \mathrm{n}=2)$. This likely accounted for why the OPTASE ${ }^{\mathrm{TM}}$ Moist Heat Mask (Group 3) appeared to have the greatest overall effect on quantity of DF using the eyelash manipulation technique. The MGDRx EyeBag® (Group 2) appeared to have the greatest effect on the overall proportion of DF $(-25.00 \%, n=4)$. However, no significant difference in proportion of DF was found between the groups at week eight $(\mathrm{K}-\mathrm{W} p=.185)$. Furthermore, no significant difference in proportion of pathogenic and non-pathogenic DF infestation was found within the groups over the eight weeks: Group 1 (Friedman's 
Table 2. Proportion of subjects with pathogenic and non-pathogenic Demodex folliculorum infestation in each treatment group, at each time point.

\begin{tabular}{|c|c|c|c|c|c|}
\hline & Time & $\begin{array}{l}\text { No Demodex \% } \\
(\mathrm{n})\end{array}$ & $\begin{array}{l}\text { Non-Pathogenic } \\
\text { Infestation \% (n) }\end{array}$ & $\begin{array}{c}\text { Pathogenic } \\
\text { Infestation \% (n) }\end{array}$ & $\begin{array}{c}\text { Overall Prevalence Demodex } \\
\text { folliculorum }\end{array}$ \\
\hline \multirow{4}{*}{$\begin{array}{l}\text { Group 1: Face Cloth } \\
\qquad(\mathrm{n}=12)\end{array}$} & Baseline & $33.33(n=4)$ & $33.33(n=4)$ & $33.33(n=4)$ & $66.67 \%(n=8)$ \\
\hline & Week Two & $33.33(n=4)$ & $41.67(n=5)$ & $25.00(n=3)$ & $66.67 \%(n=8)$ \\
\hline & Week Four & $33.33(n=4)$ & $41.67(n=5)$ & $25.00(n=3)$ & $66.67 \%(n=8)$ \\
\hline & Week Eight & $41.67(n=5)$ & $25.00(n=3)$ & $33.33(n=4)$ & $58.33 \%(n=7)$ \\
\hline Difference & (Baseline to Week Eight) & $+8.34 \%(n=1)$ & $-8.34 \%(n=-1)$ & $0.00 \%(n=0)$ & $-8.14 \%(n=-1)$ \\
\hline Group 2: MGDRx EyeBag® & Baseline & $50.00(n=8)$ & $31.25(n=5)$ & $18.75(n=3)$ & $50.00 \%(n=8)$ \\
\hline \multirow[t]{3}{*}{$(n=16)$} & Week Two & $62.50(n=10)$ & $12.50(n=2)$ & $25.00(n=4)$ & $37.50 \%(n=6)$ \\
\hline & Week Four & $62.50(n=10)$ & $25.00(n=4)$ & $12.50(n=2)$ & $37.50 \%(n=6)$ \\
\hline & Week Eight & $75.00(n=12)$ & $12.50(n=2)$ & $12.50(n=2)$ & $25.00 \%(n=4)$ \\
\hline Difference & (Baseline to Week Eight) & $+25.00 \%(n=4)$ & $-18.75 \%(n=-3)$ & $-6.25 \%(n=-1)$ & $-25.00 \%(n=-4)$ \\
\hline Group 3: Optase ${ }^{\mathrm{TM}}$ Moist & Baseline & $42.86(n=6)$ & $28.57(n=4)$ & $28.57(n=4)$ & $57.14 \%(n=8)$ \\
\hline Heat Mask & Week Two & $50.00(n=7)$ & $35.71(n=5)$ & $14.29(n=2)$ & $50.00 \%(n=7)$ \\
\hline \multirow[t]{2}{*}{$(n=14)$} & Week Four & $50.00(n=7)$ & $28.57(n=4)$ & $21.43(n=3)$ & $50.00 \%(n=7)$ \\
\hline & Week Eight & $50.00(n=7)$ & $35.71(n=5)$ & $14.29(n=2)$ & $50.00 \%(n=7)$ \\
\hline Difference & (Baseline to Week Eight) & $+7.14 \%(n=1)$ & $+7.14 \%(n=1)$ & $-14.29 \%(n=-2)$ & $-7.14 \%(n=-1)$ \\
\hline
\end{tabular}

$p=.998$ ), Group 2 (Friedman's $p=.176$ ) and Group 3 (Friedman's $p=.486$ ).

\section{MGD grade}

The box plot in Figure 3 demonstrates change in MGD grade after treatment, for each group. There was a significant reduction in MGD grade with time for each treatment (Friedman's $p=.008$, Figure 3). Post hoc analysis using Wilcoxon signed ranks test pairwise comparisons, with adjusted Bonferroni corrected alpha level, revealed significant improvements from baseline to week eight in Group 2 (median: 2, IQR: 1-2 versus median: 1 , IQR: $0-2$; WSR $p=.008$ ), and improvements from baseline to week eight (IQR: $1-2$ versus median: 1, IQR: $0-1$; WSR $p=.002$ ) and week two to week eight (median: 1, IQR: 1-2 versus median: 1, IQR: 0-1; WSR $p=.003$ ) in Group 3. There was no significant difference found between the treatments at any time point over the eight weeks ( $\mathrm{K}-\mathrm{W} p>.05)$.

\section{Modified OSDI symptoms}

Figure 4 illustrates a box plot of the change in modified OSDI symptom score for each treatment group over time. There was a significant reduction in OSDI symptom score with time for each treatment (repeated ANOVA $p=.038, p=.023$ and $p=.022$ for Groups 1-3 respectively, Table 3). As can be seen from Figure 4, the greatest reduction in symptoms appears to be in Group 2 and Group 3. Post hoc analysis with Bonferroni pair-wise comparison revealed; only the reduction in symptoms from baseline to week two and baseline to week eight ( $p=.033$ and $p=.008$ respectively) in Group 2, and reduction in symptoms from baseline to week two and baseline to week eight $(p=.013$ and $p=.047$ respectively) in Group 3, were found to be significant (Figure 4). There was no significant difference in modified OSDI symptom score between the treatments at any time point over the eight weeks (ANOVA $p>.05$, Table 3 ).

\section{Clinical signs of dry eye}

Figure 5 illustrates a box plot of the change in osmolarity values for each group at each visit. There was a significant reduction in osmolarity for subjects in Group 3 over the eight weeks (repeated ANOVA $p=.014$, Table 3 ). Bonferroni posthoc analysis showed that this was significant from baseline to week four only ( $p=.017$, Figure 5 ). There was no significant change in osmolarity detected for subjects in Groups 1 and 2 at any stage over the eight weeks (repeated ANOVA $p>.05$, Table 3). Overall, repeated measures of ANOVA taking treatment into consideration as a between subjects' factor, showed no significant change in osmolarity overtime $(p=.107)$.

The presence of tear film instability, with an inter-eye difference of $\geq 8 \mathrm{mOsm} / \mathrm{L}$, was also measured for each subject (Table 3). At baseline, the presence of tear film instability for each group was as follows: Group 1-83.33\%, Group 2-40.00\% and Group 3-71.44\%. After eight weeks tear film instability had reduced in Group 1 (41.67\%) and Group 3 (64.22\%) but had increased slightly in Group $2(60.00 \%)$. None of these changes were found to be significant within each group over time (Friedmans $p>.05$ ) or between each group at any point in time $(\mathrm{K}-\mathrm{W} p>.05)$.

There was a significant reduction in ocular surface staining over time for each treatment (Friedman's $p=.043, p=.007$ and $p=.035$ for Groups 1-3 respectively, Table 3). Post hoc analysis using Wilcoxon signed ranks test pairwise comparisons, with Bonferroni corrected alpha level, revealed; only a reduction in staining from week two to week eight in Group 2 (mean: 1.71, range 0-8; median: 1, IQR: $0-2$ versus mean:0.33, range $0-2$; median: 0, IQR: $0-0.75$; WSR $p=.006$ ), and week two to week eight in Group 3 (mean: 1.77, range 0-9; median: 1, IQR: $0-3.25$ versus mean:0.50, range $0-4$; median: 0 , IQR: $0-0.75$; WSR $p=.008$ ), were found to be significant. There was no significant difference in ocular surface staining between the treatments at any time point over the eight weeks (K-W $p>.05)$.

Mean NITBUT and Schirmer I scores for each treatment group, at each time point, can be seen in Table 3. There was no significant change in NITBUT, or Schirmer I score, over time in each group (Friedman's $p>.05$ ), or between treatments (K-W $p>.05)$.

\section{Discussion}

Demodex brevis is most commonly associated with MGD, however, it has been established in the literature that DF and MGD are 


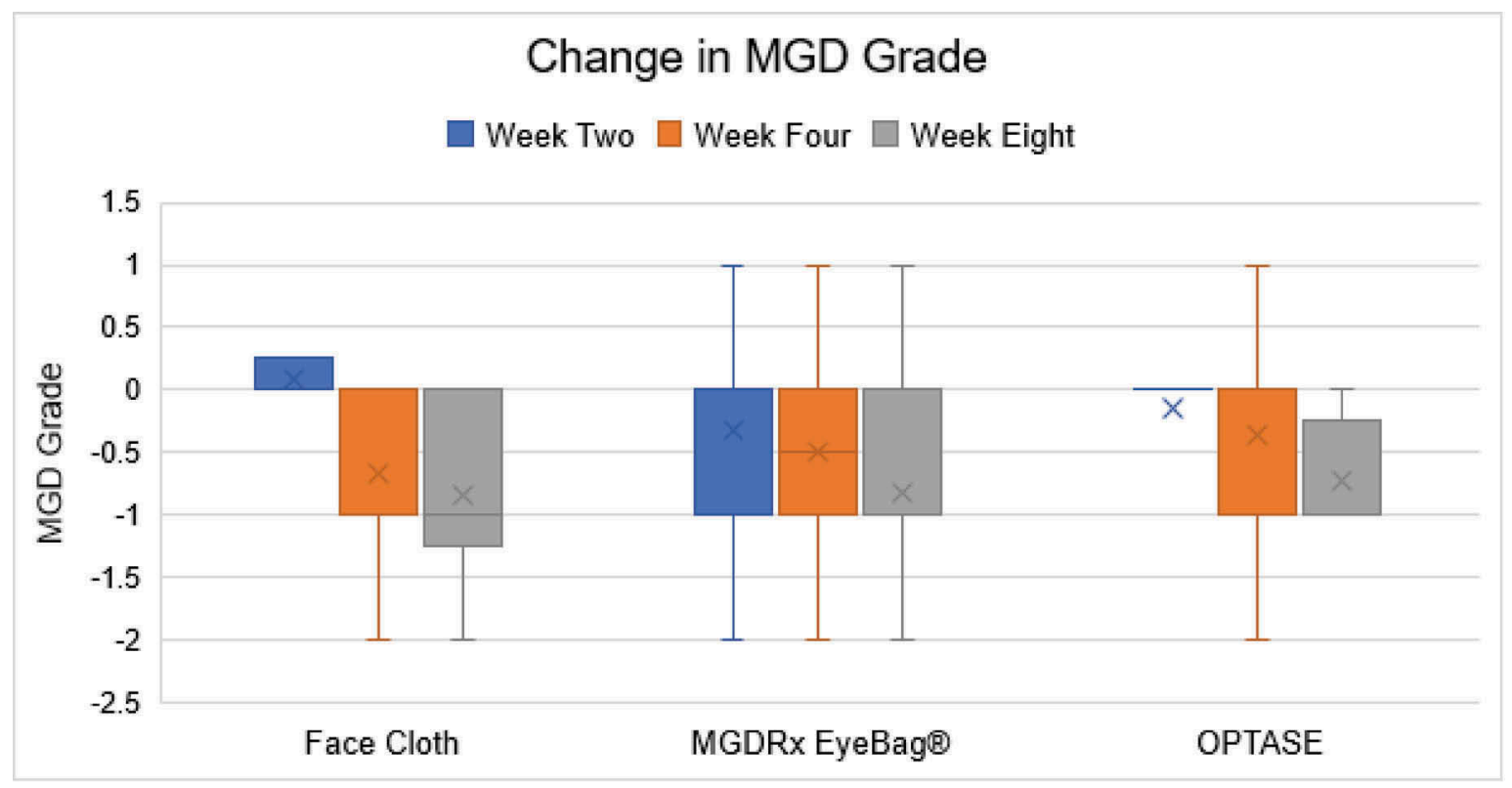

\begin{tabular}{|c|c|c|c|c|}
\hline & & Face Cloth & $\begin{array}{c}\text { MGDRx } \\
\text { EyeBag® }\end{array}$ & $\begin{array}{c}\text { OPTASE } \\
\text { Heat Mask }\end{array}$ \\
\hline \multirow{3}{*}{ Median (IQR) } & W2 & $0(0-0.75)$ & $0(-1-0)$ & $0(0-0)$ \\
\cline { 2 - 5 } & W4 & $-1(-1-0)$ & $-0.5(-1-0)$ & $0(-1-0)$ \\
\cline { 2 - 5 } & W8 & $-1(-1.75-0)$ & $-1(-1-0)$ & $-1(-1-0)$ \\
\hline p value & & $\mathrm{F} \mathbf{p}=\mathbf{0 . 0 0 8 ^ { * }}$ & $\mathrm{F} \mathbf{p}=\mathbf{0 . 0 0 2 ^ { * }}$ & $\mathrm{F} \mathbf{p}=\mathbf{0 . 0 0 2 ^ { * }}$ \\
\hline
\end{tabular}

\begin{tabular}{|c|c|c|c|}
\hline Post - hoc & Face Cloth & MGDRx EyeBag ${ }^{\circledR}$ & $\begin{array}{c}\text { OPTASETM Moist } \\
\text { Heat Mask }\end{array}$ \\
\hline BL -W2 & 0.414 & 0.527 & 1.000 \\
\hline BL - W4 & 0.035 & 0.035 & 0.180 \\
\hline BL - W8 & 0.030 & $\mathbf{0 . 0 0 8}^{*}$ & $\mathbf{0 . 0 0 2}^{*}$ \\
\hline W2 - W4 & 0.021 & 0.025 & 0.414 \\
\hline W2 - W8 & 0.018 & 0.024 & $\mathbf{0 . 0 0 3}^{*}$ \\
\hline W4 - W8 & 0.414 & 0.157 & 0.058 \\
\hline
\end{tabular}

$X$ represents the mean change in MGD Grade. Post - hoc analysis: Wilcoxon signed ranks test pairwise comparison with Bonferroni adjusted correction applied ( $\alpha \leq 0.008$ significant). BL: Baseline, W2: Week Two, W4: Week 4, W8: Week Eight. *Significant results highlighted in bold.

Figure 3. Box plot illustrating change in MGD Grade after two, four, and eight weeks with each treatment.

also connected. ${ }^{11,24,25}$ At present lid scrubs are the main treatment recommended for Demodex blepharitis, ${ }^{56}$ and warm compress therapy the mainstay treatment for MGD. ${ }^{29}$ The potential therapeutic effect of heat for treating DF infestation has been previously shown using intense pulsed light therapy. ${ }^{34}$ However, to the best of the authors' knowledge, this is the first study to investigate the effect of heat therapy using warm compresses on DF infestation. This study has demonstrated that the use of OPTASE ${ }^{\mathrm{TM}}$ Moist Heat Mask may have a double therapeutic effect, treating MGD and reducing DF in combination. Over the eight weeks, moist heat therapy from the OPTASE ${ }^{\mathrm{TM}}$ Moist Heat Mask significantly reduced the quantity of DF detected using the eyelash rotation technique. No significant change was noted with the moist heat from the warm face cloth, or the dry heat from the MGDRx
EyeBag®. The reason for this is unknown; it may be related to the compresses ability to achieve a higher treatment temperature. For the warm compresses to be effective at treating MGD, they need to heat the inner eyelid to a temperature of $\geq 40^{\circ} \mathrm{C}^{31}$ Manufacturers of OPTASE ${ }^{\mathrm{TM}}$ Moist Heat Mask report temperatures from $50^{\circ} \mathrm{C}$ to $41^{\circ} \mathrm{C}$ for the 10-minute duration of therapy. ${ }^{57}$ By comparison, the MGDRx EyeBag $®$ only achieves temperatures of $46^{\circ} \mathrm{C}$ dropping to $39^{\circ} \mathrm{C}$ after 5 minutes. ${ }^{58}$ Likewise, the warm face cloth does not retain its heat for longer than two minutes without needing to be re-heated in the cooled boiled water. ${ }^{31,54}$ This could affect the overall therapeutic temperature achieved by the face cloth with respect to treating DF infestation.

While the MGDRx EyeBag® did not appear to have a significant impact on DF quantity, the proportion of DF 
Table 3. Dry eye parameters; Modified OSDI symptom score (mean \pm SD), Osmolarity (mean \pm SD), tear film instability (\%), NITBUT (median and IQR), ocular surface staining (median and IQR), Schirmer I (median and IQR), before and after treatment.

\begin{tabular}{|c|c|c|c|c|c|}
\hline Variable & Time & Group 1 & Group 2 & Group 3 & \\
\hline Modified OSDI Score (0-100) & $\begin{array}{l}\mathrm{BL} \\
\text { W2 } \\
\text { W4 } \\
\text { W8 }\end{array}$ & $\begin{array}{c}24.21 \pm 15.27 \\
22.05 \pm 15.29 \\
15.71 \pm 7.86 \\
15.14 \pm 12.75 \\
\mathbf{R A} \boldsymbol{p}=\mathbf{. 0 3 8 *}\end{array}$ & $\begin{array}{l}39.81 \pm 23.21 \\
27.40 \pm 19.29 \\
23.03 \pm 19.55 \\
16.67 \pm 13.07 \\
\mathbf{R A}^{2}=\mathbf{0 2 3}\end{array}$ & $\begin{array}{l}39.01 \pm 20.34 \\
24.70 \pm 18.87 \\
23.65 \pm 19.89 \\
26.52 \pm 17.15 \\
\text { RA } \boldsymbol{p}=. \mathbf{0 2 2}\end{array}$ & $\begin{array}{l}{ }_{A} p=.123 \\
A_{p}=.688 \\
A_{p}=.412 \\
A_{p}=.098\end{array}$ \\
\hline Osmolarity (mOsm/L) & $\begin{array}{l}\text { BL } \\
\text { W2 } \\
\text { W4 } \\
\text { W8 }\end{array}$ & $\begin{array}{c}304.17 \pm 18.10 \\
301.27 \pm 17.47 \\
303.92 \pm 18.88 \\
305.50 \pm 18.82 \\
\operatorname{RA} p=.596\end{array}$ & $\begin{array}{c}303.53 \pm 9.55 \\
304.71 \pm 12.98 \\
299.93 \pm 13.68 \\
303.07 \pm 11.91 \\
\text { RA } p=.863\end{array}$ & $\begin{array}{c}318.86 \pm 13.44 \\
310.27 \pm 16.46 \\
305.92 \pm 14.69 \\
312.86 \pm 13.37 \\
\text { RA } \boldsymbol{p}=\mathbf{. 0 1 4}\end{array}$ & $\begin{array}{l}A_{p}=.008^{*} \\
A_{p}=.413 \\
A_{p}=.688 \\
A_{p}=.194\end{array}$ \\
\hline $\begin{array}{l}\text { Staining } \\
(0-15)\end{array}$ & $\begin{array}{l}\text { BL } \\
\text { W2 } \\
\text { W4 } \\
\text { W8 }\end{array}$ & $\begin{array}{c}1 \text { (IQR: } 0-2 \text { ) } \\
0 \text { (IQR: } 0-2 \text { ) } \\
0 \text { (IQR: } 0-0.75) \\
0 \text { (IQR: } 0-0.75) \\
\text { F } p=\mathbf{0 4 3}^{*}\end{array}$ & $\begin{array}{c}1 \text { (IQR: } 0-1.75) \\
1 \text { (IQR: } 0-2 \text { ) } \\
0 \text { (IQR: } 0-1) \\
0 \text { (IQR: } 0-0.75) \\
\text { F } p=\mathbf{0 0 7}^{*}\end{array}$ & $\begin{array}{c}0 \text { (IQR: } 0-2) \\
1 \text { (IQR: } 0-3.25) \\
0 \text { (IQR: } 0-1) \\
0 \text { (IQR: } 0-0.75) \\
\mathbf{F}=.035^{*}\end{array}$ & $\begin{aligned} \kappa w_{p} & =.978 \\
\kappa w_{p} & =.030^{*} \\
k w_{p} & =.720 \\
k w_{p} & =.953\end{aligned}$ \\
\hline NITBUT (secs) & W8 & $\begin{array}{c}\text { (IQR: } 2.83-11.10) \\
4.14 \\
(\text { IQR: } 3.14-9.46) \\
p=.954\end{array}$ & $\begin{array}{c}\text { (IQR: } 2.64-8.21) \\
6.78 \\
\text { (IQR: } 3.56-14.87) \\
F=.870\end{array}$ & $\begin{array}{c}\text { (IQR: } 2.60-6.90) \\
5.13 \\
\text { (IQR: } 2.97-5.13) \\
\quad p=.600\end{array}$ & $\mathrm{kw}_{\mathrm{p}}=.337$ \\
\hline \multirow[t]{4}{*}{ Schirmer (mm/5min) } & $\mathrm{BL}$ & $\begin{array}{c}10.50 \\
\text { (IQR: } 8.25-19.00)\end{array}$ & $\begin{array}{c}32 \\
\text { (IQR: } 11-35)\end{array}$ & $\begin{array}{c}21.50 \\
\text { (IQR: } 5.25-28.75)\end{array}$ & $\mathrm{kW}_{\mathrm{p}}=.248$ \\
\hline & W2 & $\begin{array}{c}8 \\
\text { (IQR: } 5.25-15.25)\end{array}$ & $\begin{array}{c}18 \\
\text { (IQR: } 11-35)\end{array}$ & $\begin{array}{c}13.50 \\
\text { (IQR: } 6.25-25.25)\end{array}$ & ${ }^{k W} p=.064$ \\
\hline & W4 & $\begin{array}{c}13.50 \\
\text { (IQR: } 5.75-17.75)\end{array}$ & $\begin{array}{c}28 \\
\text { (IQR: 7-35) }\end{array}$ & $\begin{array}{c}13 \\
\text { (IQR: } 7-27.75)\end{array}$ & ${ }^{k W} p=.673$ \\
\hline & W8 & $\begin{array}{c}8.00 \\
(\mathrm{IQR}: 8-13.75) \\
\mathrm{F}=.209\end{array}$ & $\begin{array}{c}20.0 \\
\text { (IQR: } 15-32) \\
\mathrm{F} p=.934\end{array}$ & $\begin{array}{c}17.00 \\
(\mathrm{IQR}: 6-17) \\
\mathrm{F}=.570\end{array}$ & ${ }^{k W} p=.065$ \\
\hline
\end{tabular}

BL: Baseline, W2: Week Two, W4: Week Four, W8: Week Eight. Statistical Tests Applied: ${ }^{\mathrm{A}}$ ANOVA, ${ }^{\mathrm{RA}}$ Repeated Measures ANOVA, ${ }^{\mathrm{KW}}$ Kruskal-Wallis, ${ }^{\mathrm{F}}$ Friedman's, ${ }^{\mathrm{X} 2}$ Chi-squared, CQ Cochran's Q. a = 0.05 significant.

*Significant results highlighted in bold.

infestation did improve considerably (by 25\%) in Group 2. Although there was no significant difference in the mean quantity of $\mathrm{DF}$ at baseline between the three treatment groups; Group 2 had the lowest quantity and lowest proportion of subjects with pathogenic infestation of the three groups at baseline. Therefore, the authors believe that these lower numbers of DF within Group 2 affected the compresses ability to demonstrate significant changes over time. A posthoc power calculation was conducted on the data, and a low power ( $1-\beta=0.26)$ was detected; which would have affected the power and significance of the results. If one was to assume baseline values were equal, then data at week eight may show that both the MGDRx EyeBag® and OPTASE ${ }^{\mathrm{TM}}$ Moist Heat Mask had similar quantities, potentially even favouring the MGDRx EyeBag®; suggesting that the MGDRx EyeBag® also had similar therapeutic abilities for treating DF blepharitis. However, due to the limitations outlined above, it is not currently possible to directly compare the efficacy of OPTASE $^{\mathrm{TM}}$ Moist Heat Mask to the MGDRx EyeBag® from the study results. Future research that focusses on pathogenic infestation (to ensure higher quantities of DF per subject), would help reduce any limitations caused by lower numbers of DF.

Similarly, no significant change was detected in quantity of DF using the traditional modified Coston method, ${ }^{48}$ for any of the warm compresses studied. As can be seen from the current study, the quantity of DF detected whilst rotating the eyelash in-situ was significantly greater than that observed on microscopic examination (paired t-test $p=.014, p=.015$ and $p=.007$ for baseline, week four and week eight respectively). No significant difference was observed in both techniques in week two, where overall a low quantity of DF was detected. The authors postulate that the low quantity of DF detected in week two may be as a result of subjects using the warm compresses for 10 minutes twice a day. After two weeks, subjects reduced treatment time to 10 minutes once a day, and the quantities of DF appeared to increase again slightly. Thus, suggesting a direct correlation between length of heat therapy and therapeutic effect. The authors speculate that the lack of significant results found using the modified Coston method was due to the limitations of the method. Undercounting, results in reduced quantities of DF, which affects the compresses ability to demonstrate change, as outlined previously.

Hyperosmolarity is considered one of the hallmark signs of dry eye disease and ocular surface inflammation..$^{59}$ In MGD, availability of meibum to the ocular surface is compromised either through reduced secretion (possibly due to poor expressibility, or severe meibomian gland dropout), or a poor quality secretion; thus causing increased tear 


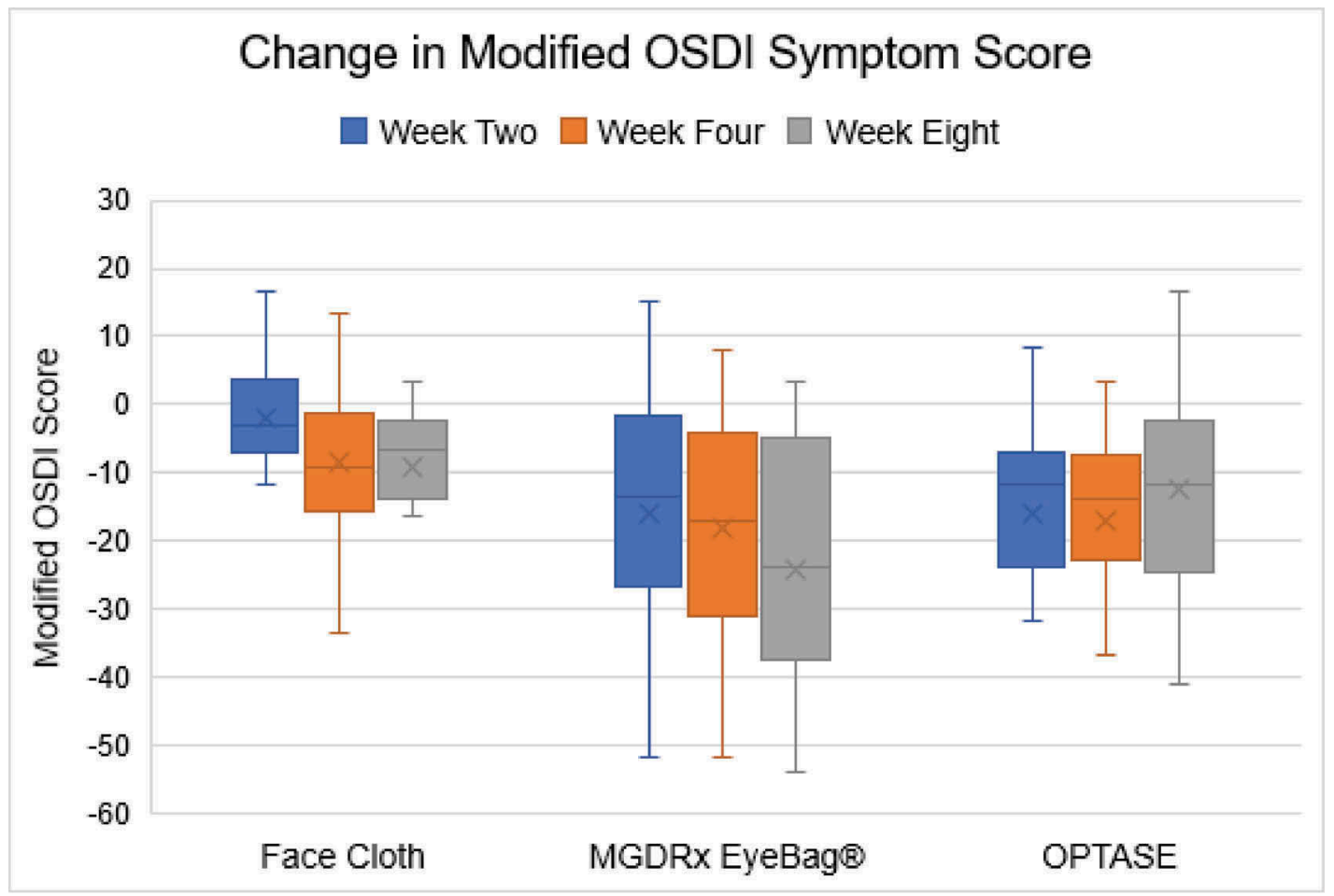

\begin{tabular}{|c|c|c|c|}
\hline Post - hoc & Face Cloth & $\begin{array}{c}\text { MGDRx } \\
\text { EyeBag® }\end{array}$ & $\begin{array}{c}\text { OPTASETM Moist } \\
\text { Heat Mask }\end{array}$ \\
\hline BL -W2 & 1.000 & $\mathbf{0 . 0 3 3}^{*}$ & $\mathbf{0 . 0 1 3}^{*}$ \\
\hline BL -W4 & 0.266 & 0.078 & 0.064 \\
\hline BL - W8 & 0.054 & $\mathbf{0 . 0 0 8 ^ { * }}$ & $\mathbf{0 . 0 4 7 ^ { * }}$ \\
\hline W2 -W4 & 0.673 & 1.000 & 1.000 \\
\hline W2 -W8 & 0.687 & 0.100 & 1.000 \\
\hline W4 -W8 & 1.000 & 0.464 & 1.000 \\
\hline
\end{tabular}

$X$ illustrates mean change in modified OSDI score. Post - hoc analysis: Bonferroni $(\alpha \leq 0.05$ significant). BL: Baseline, W2: Week Two, W4: Week 4, W8: Week Eight. *Significant results highlighted in bold.

Figure 4. Box plot illustrating change in modified OSDI symptom score for each treatment group, at each visit.

evaporation and, one would expect, an increase in osmolarity. ${ }^{60}$ However, there are conflicting reports in the literature. ${ }^{60-63}$ In the current study, subjects in Group 3 (OPTASE $^{\mathrm{TM}}$ Moist Heat Mask) demonstrated a significant improvement in osmolarity overtime. However, subjects in Group 3 had a greater osmolarity at baseline in comparison to subjects in the other two groups, which is likely to have had an effect on the overall reduction in osmolarity values detected in Group 3. Similar to the current study, Kim et al. reported a significant improvement in osmolarity post-treatment in subjects who had a pre-treatment osmolarity of $>307 \mathrm{mOsm} / \mathrm{L}$, and no significant improvement in osmolarity post-treatment in subjects who had an osmolarity of $<307 \mathrm{mOsm} / \mathrm{L}$ pre-treatement. ${ }^{64}$ The authors concluded that heat treatment with a thermal pulsation device was effective at improving osmolarity in subjects with abnormal osmolarity values, but did not have an effect on those with normal tear osmolarity. ${ }^{64}$ By comparison, Godin et al. found that treatment of MGD with a thermal pulsation device on a cohort of subjects with Sjogrens syndrome caused an initial increase in osmolarity two months after treatment (305.2 vs. $315.6, p=.026$ ), but no significant increase one year after treatment (305.2 vs $311.0, p=.86) .{ }^{61}$ Group 1 and Group 2 in the current study had similar low pretreatment osmolarity values. However, neither the heat therapy from the warm face cloth or the MGDRx EyeBag® caused an increase in osmolarity values after two months of treatment. Giannaccare et al. analysed the performance of an ocular surface work-up using modern automated noninvasive measurements for diagnosing MGD. ${ }^{62}$ The authors looked at non-invasive methods such as NITBUT, osmolarity, lipid layer thickness and non-contact meibography and 


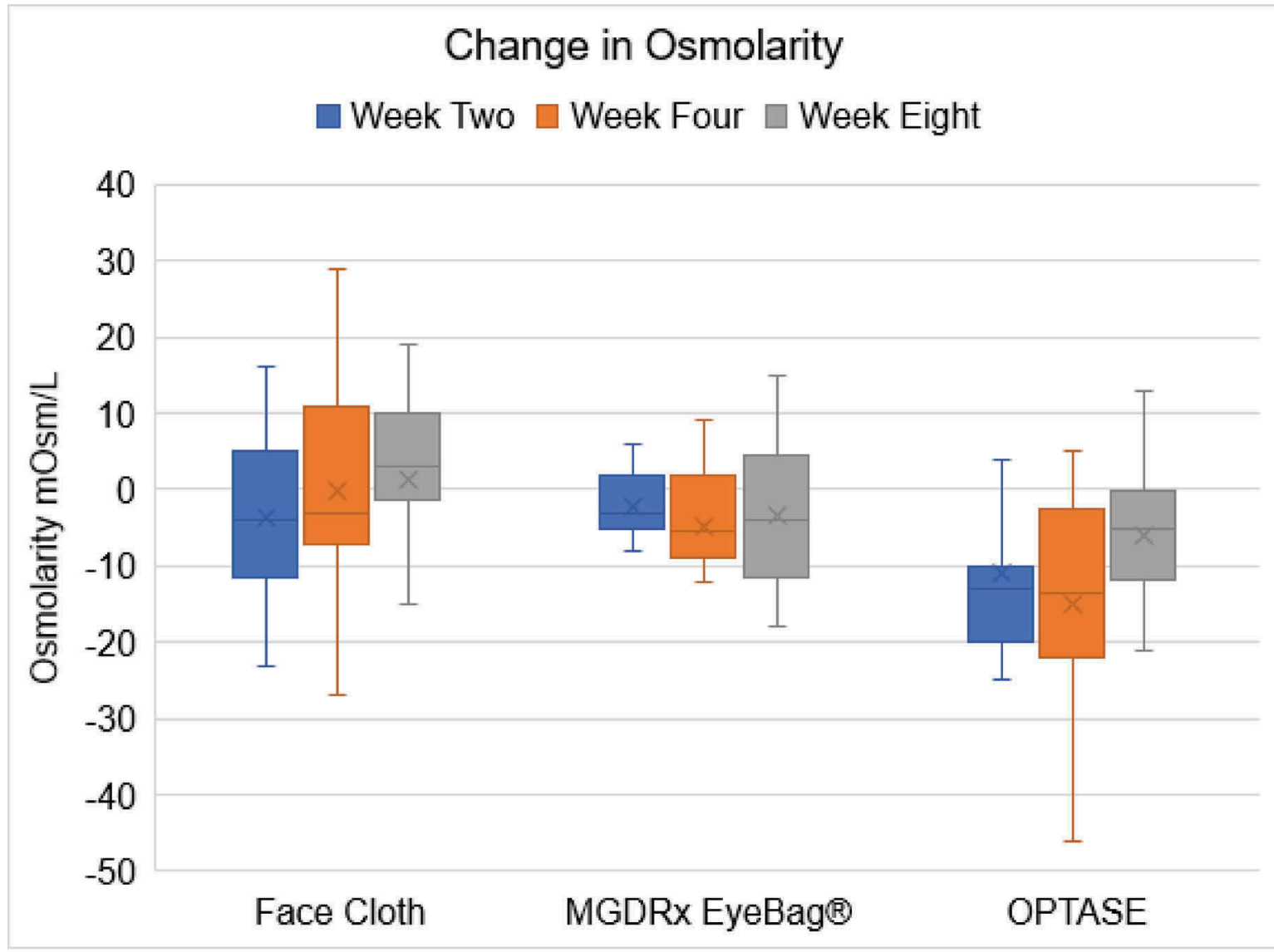

\begin{tabular}{|c|c|c|c|}
\hline Post - hoc & Face Cloth & MGDRx EyeBag® & $\begin{array}{c}\text { OPTASETM Moist } \\
\text { Heat Mask }\end{array}$ \\
\hline BL -W2 & 1.000 & 1.000 & 0.220 \\
\hline BL -W4 & 1.000 & 1.000 & $\mathbf{0 . 0 1 7 ^ { * }}$ \\
\hline BL - W8 & 1.000 & 1.000 & 0.980 \\
\hline W2 - W4 & 1.000 & 1.000 & 1.000 \\
\hline W2 - W8 & 1.000 & 1.000 & 1.000 \\
\hline W4 -W8 & 1.000 & 1.000 & 0.699 \\
\hline
\end{tabular}

$X$ denotes mean change in osmolarity. Post - hoc analysis: Bonferroni ( $\alpha \leq 0.05$ significant). BL: Baseline, W2: Week Two, W4: Week 4, W8: Week Eight. *Significant results highlighted in bold.

Figure 5. Box plot illustrating change in osmolarity values for each treatment group at each visit.

found significant differences between MGD subjects and controls for NITBUT, OSDI symptom score and meibomian gland loss. ${ }^{62}$ However, they found no significant difference in osmolarity values between the two groups. ${ }^{62}$ Similar to the current study, and the study by Godin et al., ${ }^{61}$ Giannaccare et al. found a low mean osmolarity value among their MGD subjects $(303.5 \pm 9.8 \mathrm{mOsm} / \mathrm{L}){ }^{62}$ The lack of significance found in the current and previous studies, may be related to the low osmolarity found among MGD patients. ${ }^{61,62}$ It has been proposed that MGD disease may not cause an increase in tear osmolarity, as the disease alone may not be sufficient enough to alter the homeostatic control in many subjects. ${ }^{62,63}$ In the current study, the authors have been unable to find an explanation for the higher osmolarity values observed in Group 3. Subjects in Group 3 were not significantly older, or more symptomatic, and did not have a significantly greater quantity of DF, MGD, ocular surface staining, or reduced TBUT or tear secretion at baseline. Furthermore, repeated measures of ANOVA, with treatment as a between subjects' factor, showed no significant change in osmolarity overtime. Therefore, the authors propose that the increased osmolarity value observed at baseline in Group 3 may be happenstance with regards to the current study.

Heat therapy has been shown to increase the availability of meibum to the tear film and ocular surface, helping to improve the stability of the tears, and thus, increase tear break-up time (TBUT) ${ }^{54,58,65-67}$ In the current study, although changes were 
not significant, the MGDRx EyeBag ${ }^{\circledR}$, the dry warming device, demonstrated the greater increase in NITBUT of the three compresses examined. These results are in keeping with Arita et al. who found that only dry warming devices were able to significantly improve the oily tear film layer, and therefore TBUT. ${ }^{65}$ In the same study, no improvement was observed after the repeated use of a hot towel compress, and the authors' concluded that moisture on the surface of the eyelid skin may possibly give rise to evaporative cooling; thus limiting the favourable effects of warming. ${ }^{65}$ None of the compresses used in the current study demonstrated significant increases in NITBUT. This lack of significance may be attributed to the differences in measurement techniques and timings of measurements posttreatment. In previous studies, TBUT has been measured invasively using fluorescein, ${ }^{67,68}$ or non-invasively using a TearScope Plus. ${ }^{58,66}$ The invasive nature of the fluorescein dye has been shown to alter the tear film and affect the natural TBUT. ${ }^{69,70}$ Although the TearScope Plus is a non-invasive method, it is a subjective measurement, and relies on the examiner observing the first noticeable break in the fine line pattern. Conversely, in the current study NITBUT was measured using the automated Medmont E300 Corneal Topographer, which has demonstrated good repeatability with a high sensitivity and specificity for diagnosing moderate to severe dry eye disease..$^{53}$ Thus, all measurements were objectively taken by the instrument, which is more sensitive to small tear film instabilities. Furthermore, in previous studies, many TBUT measurements have been taken immediately after, 5-10 minutes after, or up to 1 hour after heat therapy has been applied. ${ }^{58,65,66}$ In the current study, subjects applied the warm compresses at home in the evenings. Therefore, there was a much greater time gap between last warm compress and time of measurement. Although immediate effect of treatment has not been established in the current study, the results do demonstrate the 'real' effect of each treatment on subjects' tear film and ocular surface.

A limitation of the current study was that subjects were applying the heat therapy at home. As a result, it was not possible to measure the temperature of the compress each time it was used. Insufficient lid warming of warm compresses have been noted previously in the literature. ${ }^{71,72}$ Although all subjects were given written instructions, it is possible that subjects may not have heated the compress sufficiently, or did not complete the full 10-minute therapy requested of them. In an attempt to monitor compliance, at each aftercare, subjects were asked to report on how many evenings and for how long they used the warm compress as instructed. The overall reported compliance at the end of the study was 83.74\%: 77.92\% warm face cloth, 86.81\% MGDRx EyeBag®, and $85.45 \%$ OPTASE $^{\mathrm{TM}}$ Moist Heat Mask. Poor compliance is a problem with examining efficacy of treatments, thus it would be preferable to have $100 \%$ compliance and treatment provided in-house by the examiner. As the warm face cloth group had the lowest compliance, it cannot be ruled out that this impacted on the ability of the compress to demonstrate any significant results for treating MGD or DF blepharitis. However, the use of warm compresses in the current study is potentially a better indicator of warm compress use in 'real world' environments.
The MGDRx EyeBag® and the OPTASE ${ }^{\mathrm{TM}}$ Moist Heat Mask significantly reduced the presence of MGD over the duration of the study. Although some improvement in MGD was seen with the warm face cloth, these changes were not found to be significant. Furthermore, the warm face cloth compress had to be re-dipped every 2 minutes, as it lost its heat quickly. Therefore, compresses that can be heated once and used for 10 minutes at a time are much more convenient for patient use. There was also a higher rate of attrition in the warm face cloth group than either of the other two groups, and overall compliance was lowest in this group. The authors feel that this was due to the inconvenient nature of the treatment. As such, if patients will not be compliant with a treatment, for example due to inconvenience, then it won't matter whether it works in a clinical lab scenario if it won't be used correctly in the 'real-world'.

The microwaveable compresses, MGDRx EyeBag® and OPTASE $^{\mathrm{TM}}$ Moist Heat Mask, exhibited a greater ability to treat MGD, reduce symptoms and reduce ocular surface staining, in comparison to the more 'traditional' warm face cloth compress. The OPTASE ${ }^{\mathrm{TM}}$ Moist Heat Mask appears to demonstrate an ability to provide dual treatment to patients with MGD and Demodex blepharitis. The MGDRx Eyebag ${ }^{\circledR}$ demonstrated a possible ability to provide dual treatment for MGD and Demodex blepharitis, however, further research is required to confirm.

\section{Acknowledgments}

The authors thank the management and staff of the National Optometry Centre, for providing use of the premises and equipment, and helping with diary management and subject recruitment.

\section{Funding}

This research was conducted as part of a self-funded post-graduate research degree.

\section{Declaration of Interests}

The materials, OPTASE ${ }^{\mathrm{TM}}$ Moist Heat Mask and MGDRx EyeBag®, were supplied by Scope Ophthalmics Ltd., Dublin. The authors do not have any financial interest in any of the products mentioned in this study. The authors report no conflicts of interest. The authors alone are responsible for the content and writing of the paper.

\section{ORCID}

Orla Murphy (D) http://orcid.org/0000-0002-5390-0892

\section{References}

1. Jester J, Nicolaides N, Smith R. Meibomian gland studies: histologic and ultrastructural investigations. Invest Ophthalmol Vis Sci. 1977;20:537-47.

2. Knop E, Knop N, Millar T, Obata H, Sullivan D. The international workshop on meibomian gland dysfunction: report of the subcomittee on anatomy, physiology and pathophysiology of the meibomian gland. Invest Ophthalmol Vis Sci. 2011;52:1938-78. doi:10.1167/iovs.10-6997c. 
3. Holly F, Lemp M. Tear physiology and dry eyes. Surv Ophthalmol. 1977;22:69-87. doi:10.1016/0039-6257(77)90087-X.

4. Bron A, Tiffany J, Gouveia S, Yokoi N, Voon L. Functional aspects of the tear film lipid layer. Exp Eye Res. 2004;78:347-60. doi:10.1016/j.exer.2003.09.019.

5. Jeyalatha M, Qu Y, Liu Z, Ou S, He X, Bu J, Li S, Reinach P, Liu Z, Li W. Function of meibomian gland: contribution of proteins. Exp Eye Res. 2017;163:29-36. doi:10.1016/j.exer.2017.06.009.

6. Foulks GN. The correlation between the tear film lipid layer and dry eye disease. Surv Ophthalmol. 2007;52:369-74. doi:10.1016/j. survophthal.2007.04.009.

7. Nichols KK, Foulks GN, Bron AJ, Glasgow BJ, Dogru M, Tsubota K, Lemp MA, Sullivan DA. The international workshop on meibomian gland dysfunction: executive summary. Invest Ophthalmol Vis Sci. 2011;52:1922. doi:10.1167/iovs.10-6997a.

8. Mathers WD, Shields WJ, Sachdev MS, Petroll WM, Jester JV. Meibomian gland dysfunction in chronic blepharitis. Cornea. 1991;10:277-85. doi:10.1097/00003226-199107000-00001.

9. McCulley JP, Shine WE. Eyelid disorders: the meibomian gland, blepharitis, and contact lenses. Eye Contact Lens. 2003;29:S93S95; discussion S115-S118, S192-S194. doi:10.1097/00140068200301001-00026.

10. Kheirkhah A, Casas V, Li W, Raju VK, Tseng SCG. Corneal manifestations of ocular demodex infestation. Am J Ophthalmol. 2007;143:743-49. doi:10.1016/j.ajo.2007.01.054.

11. Czepita D, Kuźna-Grygiel W, Czepita M, Grobelny A. Demodex folliculorum and Demodex brevis as a cause of chronic marginal blepharitis. Ann Acad Med Stetin. 2007;53:63-67.

12. Coston TO. Demodex folliculorum blepharitis. Trans Am Ophthalmol Soc. 1967;65:361-92.

13. Desch C, Nutting WB. Demodex folliculorum (Simon) and D. brevis Akbulatova of man: redescription and reevaluation. J Parasitol. 1972;58:169. doi:10.2307/3278267.

14. English F. Demodex folliculorum and oedema of the eyelash. $\mathrm{Br}$ J Ophthalmol. 1971;55:742-46. doi:10.1136/bjo.55.11.742.

15. Czepita D, Kuźna-Grygiel W, Kosik-Bogacka D. Demodex as an etiological factor in chronic blepharitis. Klin Oczna. 2005;107:722-24.

16. Liu J, Sheha H, Tseng SCG. Pathogenic role of Demodex mites in blepharitis. Curr Opin Allergy Clin Immunol. 2010;10:505-10. doi:10.1097/ACI.0b013e32833df9f4.

17. Kabataş N, Doğan AŞ, Kabataş EU, Acar M, Biçer T, Gürdal C. The effect of demodex infestation on blepharitis and the ocular symptoms. Eye Contact Lens. 2017;43:64-67. doi:10.1097/ ICL.0000000000000234.

18. Sędzikowska A, Osęka M, Skopiński P. The impact of age, sex, blepharitis, rosacea and rheumatoid arthritis on Demodex mite infection. Arch Med Sci. 2018;14:353-56. doi:10.5114/aoms.2016.60663.

19. Zhao Y-E, Wu L-P, Hu L, Xu J-R. Association of blepharitis with Demodex : a meta-analysis. Ophthalmic Epidemiol. 2012;19:95-102. doi:10.3109/09286586.2011.642052.

20. English FP, Nutting WB. Demodicosis of ophthalmic concern. Am J Ophthalmol. 1981;91:362-72. doi:10.1016/0002-9394(81)90291-9.

21. Lacey N, Kavanagh K, Tseng S. Under the lash: demodex mites in human diseases. Biochem (Lond). 2009;31:2-6.

22. Randon $M$, Liang $H$, El Hamdaoui $M$, Tahiri R, Batellier L, Denoyer A, Labbé A, Baudouin C. In vivo confocal microscopy as a novel and reliable tool for the diagnosis of Demodex eyelid infestation. $\mathrm{Br} \mathrm{J}$ Ophthalmol. 2015;99:336-41. doi:10.1136/ bjophthalmol-2014-305671.

23. Liang L, Liu Y, Ding X, Ke H, Chen C, Tseng SCG. Significant correlation between meibomian gland dysfunction and keratitis in young patients with Demodex brevis infestation. Br J Ophthalmol. 2017 October;11:bjophthalmol-2017-310302.

24. Zhang X-B, Ding Y-H HW. The association between demodex infestation and ocular surface manifestations in meibomian gland dysfunction. Int J Ophthalmol. 2018;11:589-92. doi:10.18240/ ijo.2018.04.08.

25. de Venecia AB, Lim Bon Siong R. Demodex sp. infestation in anterior blepharitis, meibomian-gland dysfunction, and mixed blepharitis. Philipp J Ophthalmol. 2011;36:15-22.
26. Gao YY, Di Pascuale MA, Elizondo A, Tseng SC. Clinical treatment of ocular demodecosis by lid scrub with tea tree oil. Cornea. 2007;26:136-43. doi:10.1097/01.ico.0000244870.62384.79.

27. Gao YY, Xu DL, Huang LJ, Wang R, Tseng SCG. Treatment of ocular itching associated with ocular demodicosis by $5 \%$ tea tree oil ointment. Cornea. 2012;31:14-17. doi:10.1097/ICO.0b013e31820ce56c.

28. Murphy O, O’Dwyer V, Lloyd-McKernan A. The efficacy of tea tree face wash, 1,2-Octanediol and microblepharoexfoliation in treating Demodex folliculorum blepharitis. Contact Lens Anterior Eye. 2018;41:77-82. doi:10.1016/j.clae.2017.10.012.

29. Geerling G, Tauber J, Baudouin C, Goto E, Matsumoto Y, O’Brien T, Rolando M, Tsubota K, Nichols KK. The international workshop on meibomian gland dysfunction: report of the subcommittee on management and treatment of meibomian gland dysfunction. Invest Ophthalmol Vis Sci. 2011;52:2050. doi:10.1167/iovs.10-6997g.

30. McCulley JP, Shine WE. Meibomian secretions in chronic blepharitis. Boston MA: Springer; 1998. p. 319-26.

31. Blackie CA, Solomon JD, Greiner JV, Holmes M, Korb DR. Inner eyelid surface temperature as a function of warm compress methodology. Optom Vis Sci. 2008;85:675-83. doi:10.1097/ OPX.0b013e318181adef.

32. Zhao YE, Guo N, Wu LP. The effect of temperature on the viability of Demodex folliculorum and Demodex brevis. Parasitol Res. 2009;105:1623-28. doi:10.1007/s00436-009-1603-x.

33. Murakami DK, Blackie CA, Korb DR. All warm compresses are not equally efficacious. Optom Vis Sci. 2015;92:e327-33. doi:10.1097/OPX.0000000000000675.

34. Zhang X, Song N, Gong L. Therapeutic effect of intense pulsed light on ocular demodicosis. Curr Eye Res. 2018 October;44:1-7.

35. Smith G, Dart J. External eye disease. In: Jackson T, editor. Moorfields manual of ophthalmology. Missouri, MO: Mosby Elsevier; 2008. p. 109-143.

36. Lacroix Z, Léger $\mathrm{S}$, Bitton E. Ex vivo heat retention of different eyelid warming masks. Contact Lens Anterior Eye. 2015;38:152-56. doi:10.1016/j.clae.2015.01.005.

37. World Medical Association. World medical association declaration of Helsinki ethical principles for medical research involving human subjects. JAMA. 2013;310:2191-94. doi:10.1001/jama.2013.281053.

38. Spickett SG. Studies on Demodex folliculorum Simon (1842). I. Life History. Parasitology. 1961;51:181-92. doi:10.1017/S0031182000068 $58 \mathrm{X}$.

39. Tomlinson A, Bron AJ, Korb DR, Amano S, Paugh JR, Pearce EI, Yee R, Yokoi N, Arita R, Dogru M. The International Workshop on Meibomian Gland Dysfunction: report of the diagnosis subcommittee. Invest Ophthalmol Vis Sci. 2011;52:2006-49. doi:10.1167/iovs.10-6997f

40. Foulks GN. Challenges and pitfalls in clinical trials of treatments for dry eye. Ocul Surf. 2003;1:20-30. doi:10.1016/S1542-0124(12) 70004-6.

41. Wolffsohn JS, Arita R, Chalmers R, Djalilian A, Dogru M, Dumbleton K, Gupta PK, Karpecki P, Lazreg S, Pult H, et al. TFOS DEWS II diagnostic methodology report. Ocul Surf. 2017;15:539-74. doi:10.1016/j.jtos.2017.05.001.

42. Murphy O, O'Dwyer V, Lloyd-McKernan A. Demodex folliculorum: prevalence and associated symptoms in an Irish population. Int Ophthalmol. 2018 January;51:1-13.

43. Lee SH, Chun YS, Kim JH, Kim ES, Kim JC. The relationship between demodex and ocular discomfort. Invest Ophthalmol Vis Sci. 2010;51:2906-11. doi:10.1167/iovs.09-4850.

44. Lemp MA, Bron AJ, Baudouin C, Benítez Del Castillo JM, Geffen D, Tauber J, Foulks GN, Pepose JS, Sullivan BD. Tear Osmolarity in the Diagnosis and Management of Dry Eye Disease. Am J Ophthalmol. 2011;151:792-798.e1.

45. Jacobi C, Jacobi A, Kruse FE, Cursiefen C. Tear film osmolarity measurements in dry eye disease using electrical impedance technology. Cornea. 2011;30:1289-92. doi:10.1097/ICO.0b013e3182 1 de383.

46. Bron AJ, Evans VE, Smith JA. Grading Of corneal and conjunctival staining in the context of other dry eye tests. Cornea. 2003;22:640-50. doi:10.1097/00003226-200310000-00008. 
47. Serin D, Karsloğlu Ş, Kyan A, Alagöz G. A simple approach to the repeatability of the schirmer test without anesthesia. Cornea. 2007;26:903-06. doi:10.1097/ICO.0b013e3180950083.

48. Gao YY, Di Pascuale MA, Li W, Liu DTS, Baradaran-Rafii A, Elizondo A, Kawakita T, Raju VK, Tseng SCG. High prevalence of Demodex in eyelashes with cylindrical dandruff. Invest Ophthalmol Vis Sci. 2005;46:3089-94. doi:10.1167/ iovs.05-0275.

49. Mastrota KM. Method to identify demodex in the eyelash follicle without epilation. Optom Vis Sci. 2013;90:e172-4. doi:10.1097/ OPX.0b013e318294c2c0.

50. Murphy O, O' Dwyer V, Lloyd-McKernan A. The clinical use of eyelash manipulation in the diagnosis of Demodex folliculorum blepharitis. Eye Contact Lens J. 2019.

51. Koo TK, Li MY. A guideline of selecting and reporting intraclass correlation coefficients for reliability research. J Chiropr Med. 2016;15:155-63. doi:10.1016/j.jcm.2016.02.012.

52. Armstrong RA. Statistical guidelines for the analysis of data obtained from one or both eyes. Ophthalmic Physiol Opt. 2013;33:7-14. doi:10.1111/opo.2012.33.issue-1.

53. Downie LE. Automated tear film surface quality breakup time as a novel clinical marker for tear hyperosmolarity in dry eye disease. Invest Ophthalmol Vis Sci. 2015;56:7260. doi:10.1167/iovs.15-17772.

54. Olson MC, Korb DR, Greiner JV. Increase in tear film lipid layer thickness following treatment with warm compresses in patients with meibomian gland dysfunction. Eye Contact Lens. 2003;29:96-99. doi:10.1097/01.ICL.0000060998.20142.8D.

55. Kim H-Y. Statistical notes for clinical researchers: nonparametric statistical methods: 2. Nonparametric methods for comparing three or more groups and repeated measures. Restor Dent Endod. 2014;39:329-32. doi:10.5395/rde.2014.39.4.329.

56. The College of Optometrists. Blepharitis (Lid Margin Disease). Clin Manag Guidel 2018. London: The College of Optometrists; 2018.

57. Rogers P. A technical report for scope ophthalmics (OPTASE Moist Heat Mask). Milton Keynes, UK: Intertek Testing \& Certification Ltd.; 2016.

58. Bilkhu PS, Naroo SA, Wolffsohn JS. Effect of a commercially available warm compress on eyelid temperature and tear film in healthy eyes. Optom Vis Sci. 2013;91:1. doi:10.1097/OPX.0000000000000134.

59. Craig JP, Nichols KK, Akpek EK, Caffery B, Dua HS, Joo C-K, Liu Z, Nelson JD, Nichols JJ, Tsubota K, et al. TFOS DEWS II definition and classification report. Ocul Surf. 2017;15:276-83. doi:10.1016/j.jtos.2017.05.008.

60. McMonnies CW. Conjunctival tear layer temperature, evaporation, hyperosmolarity, inflammation, hyperemia, tissue damage, and symptoms: a review of an amplifying cascade. Curr Eye Res. 2017;42:1574-84. doi:10.1080/02713683.2017.1377261.

61. Godin MR, Stinnett SS, Gupta PK. Outcomes of thermal pulsation treatment for dry eye syndrome in patients with sjogren disease. Cornea. 2018;37:1155-58. doi:10.1097/ICO.0000000000001621.

62. Giannaccare G, Vigo L, Pellegrini M, Sebastiani S, Carones F. Ocular surface workup with automated noninvasive measurements for the diagnosis of meibomian gland dysfunction. Cornea. 2018;37:740-45. doi:10.1097/ICO.0000000000001500.

63. Potvin R, Makari S, Rapuano CJ. Tear film osmolarity and dry eye disease: a review of the literature. Clin Ophthalmol. 2015;9:2039-47. doi:10.2147/OPTH.

64. Kim MJ, Stinnett SS, Gupta PK. Effect of thermal pulsation treatment on tear film parameters in dry eye disease patients. Clin Ophthalmol. 2017;11:883-86. doi:10.2147/OPTH.

65. Arita R, Morishige N, Shirakawa R, Sato Y, Amano S. Effects of eyelid warming devices on tear film parameters in normal subjects and patients with meibomian gland dysfunction. Ocul Surf. 2015;13:321-30. doi:10.1016/j.jtos.2015.04.005.

66. Wang MTM, Jaitley Z, Lord SM, Craig JP. Comparison of self-applied heat therapy for meibomian gland dysfunction. Optom Vis Sci. 2015;92:e321-6. doi:10.1097/OPX.0000000000000601.

67. Matsumoto $\mathrm{Y}$, Dogru M, Goto E, Ishida R, Kojima T, Onguchi T, Yagi Y, Shimazaki J, Tsubota K. Efficacy of a new warm moist air device on tear functions of patients with simple meibomian gland dysfunction. Cornea. 2006;25:644-50. doi:10.1097/01.ico.0000208822.70732.25.

68. Goto E, Monden Y, Takano Y, Mori A, Shimmura S, Shimazaki J, Tsubota K. Treatment of non-inflamed obstructive meibomian gland dysfunction by an infrared warm compression device. $\mathrm{Br}$ J Ophthalmol. 2002;86:1403-07. doi:10.1136/bjo.86.12.1403.

69. Mengher LS, Bron AJ, Tonge SR, Gilbert DJ. Effect of fluorescein instillation on the pre-corneal tear film stability. Curr Eye Res. 1985;4:9-12. doi:10.3109/02713688508999961.

70. Mooi JK, Wang MTM, Lim J, Müller A, Craig JP. Minimising instilled volume reduces the impact of fluorescein on clinical measurements of tear film stability. Contact Lens Anterior Eye. 2017;40:170-74. doi:10.1016/j.clae.2017.01.004.

71. Lane SS, DuBiner HB, Epstein RJ, Ernest PH, Greiner JV, Hardten DR, Holland EJ, Lemp MA, McDonald JE, Silbert DI, et al. The lipiflow, for the treatment of meibomian gland dysfunction. Cornea. 2012;31 :396-404. doi:10.1097/ICO.0b013e318239aaea.

72. Pult H, Riede-Pult BH, Purslow C. A comparison of an eyelid-warming device to traditional compress therapy. Optom Vis Sci. 2012;89:E1035-41. doi:10.1097/OPX.0b013e31825c3479. 